\title{
Predicción de la variabilidad del rendimiento de papa a partir de la cobertura del follaje
}

\author{
de la Casa, A.; G. Ovando, L. Bressanini, J. Martínez y A. Rodríguez
}

\begin{abstract}
RESUMEN
La cobertura del follaje $(f)$ es un parámetro biofísico importante para determinar tanto la fracción de radiación fotosintéticamente activa interceptada (fRFAl), como la tasa de agua que transpira un cultivo. En cultivos de papa (Solanum tuberosum L., cv. Spunta) de ciclo otoñal se analizó en 2009 y 2010 la relevancia de $f$ y $f R F A l$, para estimar el rendimiento y evaluar la variabilidad productiva espacial en un lote del cinturón verde de la ciudad de Córdoba, Argentina. Si bien fRFA/ presentó mayor capacidad para explicar la variabilidad del rendimiento de tubérculo a cosecha, el empleo de la duración de la cobertura, $f$ acumulada hasta los 60 días de la plantación, en 2009 alcanzó un $R^{2}$ de 0,77 $(P<0,01)$, valor que aumentó a 0,95 en 2010 a los 70 días de la plantación a partir de un muestreo más intensivo. El empleo de un único valor de $f$ obtenido próximo a cobertura máxima reduce la capacidad predictiva respecto de los valores acumulados. A partir de una grilla de muestreo de 5×5, en 2009 se constató que existe un componente significativo de la variabilidad asociada a la estructura de plantación por surcos.
\end{abstract}

Palabras clave: papa, fracción de cobertura, duración de la cobertura, predicción de rendimiento, variabilidad espacial.

de la Casa, A.; G. Ovando, L. Bressanini, J. Martínez and A. Rodríguez, 2012. Predicting potato yield variability from foliage cover. Agriscientia XXIX: $15-24$

\section{SUMMARY}

The ground cover $(f)$ is an important biophysical parameter to determine both the fraction of intercepted photosynthetically active radiation (fRFAl), and the rate of water that is transpired by a crop. During the autumn/late growing seasons of 2009 and 2010, the potential of $f$ and fRFAl to estimate potato yield (Solanum tuberosum L., cv. Spunta), and to evaluate the spacial variability of production in a plot of the green belt of Cordoba, Argentina, was discussed. While $f R F A$ / showed greater ability to explain the variability of tuber yield at harvest, using ground cover duration, the $f$ accumulated until 60 days after planting reached an $R^{2}$ of $0.77(P<0.01)$ in 2009, which increased to 0.95 in 2010 70 days after planting from a more intensive sampling ( $f$ was obtained from an 
average of nine photographs taken at $2.5 \mathrm{~m}$ height). The use of a single value of $f$ obtained near maximum coverage reduces the predictive power of cumulative values. Based on a $5 \times 5$ grid sampling in 2009 it was found that there is a significant component of the variability associated with furrow planting.

Key words: potato, ground cover, duration of ground cover, yield prediction, spatial variability.

A. de la Casa; G. Ovando, L. Bressanini, J. Martínez y A. Rodríguez. Facultad de Ciencias Agropecuarias, Universidad Nacional de Córdoba, CC 509, 5000 Córdoba, Argentina. Correspondencia a: delacasa@agro.unc.edu.ar

\section{INTRODUCCIÓN}

La productividad de los lotes de papa en el cinturón verde de Córdoba, Argentina, presentan habitualmente cierto rasgo de heterogeneidad espacial, que está relacionada a múltiples factores de orden técnico (deficiencias operativas en la plantación); del ambiente edáfico (variabilidad temporal y espacial de la fertilidad física y química (de la Casa et al., 2003)); de las condiciones sanitarias del cultivo (enfermedades y plagas), como acontece frecuentemente con el tizón tardío (Van Oijen, 1991), entre otras causas. Por otra parte, en la programación e implementación de la agricultura de precisión se requiere conocer la variabilidad productiva del lote como así también identificar los principales factores que la determinan (Moran et al., 1997; Pierce \& Novak, 1999).

De acuerdo a distintas evaluaciones realizadas en papa, la tasa de intercepción de la radiación y la extensión del ciclo se presentan como factores críticos que determinan la capacidad productiva del cultivo, tanto en la producción de biomasa al comienzo como en la de tubérculos cosechados al final del ciclo (Kooman et al., 1996 a, b; de la Casa et al., 2007). Caldiz et al. (1999) integraron la información de cobertura con respecto al tiempo transcurrido entre las observaciones, como un indicador del potencial productivo de papa.

Desde otro enfoque conceptual, Steduto et al. (2009) presentaron el modelo de cultivo AquaCrop, estructurado para estimar la producción de biomasa a partir del concepto de eficiencia en el uso del agua (Stanhill, 1986; Evett \& Tolk, 2009). El modelo emplea la cobertura para estimar la tasa de transpiración y, de este modo, diferenciar el consumo de agua con respecto al gasto evaporativo no productivo, lo que confiere a este parámetro biofísico un carácter fundamental.

La fracción de radiación fotosintéticamente activa interceptada ( $f R F A l$ ) por un cultivo se determina habitualmente con un interceptómetro, instrumento que mide la radiación solar y, a partir de la relación entre el valor que incide sobre el suelo y el que recibe sobre el follaje, estima la energía disponible para el proceso de fotosíntesis. El uso de este dispositivo impone ciertas restricciones operativas; así, es necesario que las lecturas instantáneas se realicen con ángulos cenitales bajos (próximo al mediodía solar), con una proporción elevada de radiación directa (con cielo despejado) y, en particular, evitando condiciones de nubosidad variable (de la Casa et al., 2007).

Una manera alternativa para estimar fRFAl y que tiene intrínsecamente muchas menos restricciones de uso, es a partir de las mediciones de la fracción de suelo cubierto por el follaje o cobertura del follaje $(f)$. Estos valores se pueden obtener de una manera accesible y económica por medio de fotografías digitales sobre el cultivo (Steven et al., 1986). En soja, Purcell (2000) obtuvo una relación lineal que no difiere estadísticamente de la función identidad al comparar las mediciones al mediodía de ambas variables, y sugiere emplear directamente el valor de cobertura en lugar de la fracción de radiación interceptada por tratarse de una medición más sencilla de realizar. En papa, de la Casa et al. (2008) determinaron que la relación entre $f$ y fRFAl presenta cierta desviación respecto a la función identidad. Por su parte, Boyd et al. (2002) determinaron una alta correlación entre $f y$ el índice de área foliar (IAF), y no encontraron diferencias cuando emplearon $f$ como equivalente de fRFAl y la fRFAl calculada por la ley de LambertBeer a partir del $I A F$, a fin de estimar la productividad en papa.

Para efectuar un análisis de la capacidad productiva de un cultivo de papa en un lote, que pueda servir de base tanto para analizar la conveniencia de realizar el manejo a tasa variable como 
para planificar su eventual implementación, es necesario desarrollar técnicas que permitan conocer de manera anticipada la dinámica productiva de cada sector del predio y determinar los factores relacionados con la variabilidad espacial existente (Pierce \& Novak, 1999). Las técnicas de fotografía digital ofrecen una oportunidad accesible y económica para identificar de manera temprana sectores con distinta capacidad productiva potencial, como así también para realizar un análisis espacial que permita reconocer la estructura de variabilidad del lote de producción.

En razón de su capacidad para representar tanto la intercepción de la radiación como la tasa de transpiración del cultivo, $f$ puede tener un rol de indicador temprano de la capacidad productiva del cultivo de papa. Además, la facilidad de realizar su medición por medio de fotografías se puede aprovechar a los efectos de determinar el rendimiento en forma anticipada a la cosecha y determinar la variabilidad productiva de un lote.

El objetivo de este trabajo consistió en evaluar el desempeño de la cobertura del follaje como indicador temprano de la productividad en papa para cultivos de ciclo tardío. Asimismo, se propone utilizar la cobertura del follaje para analizar la variabilidad espacial de la productividad del cultivo en el lote.

\section{MATERIAL Y MÉTODOS}

El trabajo se realizó sobre cultivos de papa (Solanum tuberosum L. cv. Spunta) de ciclo otoñal o tardío, a partir de la información producida en los años 2009 y 2010. Las mediciones se realizaron en un lote de producción comercial de 9 ha ubicado en el cinturón verde de la ciudad de Córdoba, Argentina ( $31^{\circ} 30^{\prime} \mathrm{S}, 64^{\circ} 08^{\prime} \mathrm{O}, 402 \mathrm{msnm}$ ). El suelo del sector corresponde a un Haplustol éntico, serie Manfredi, limosa fina, mixta, térmica (Jarsún et al., 2006), que no presenta limitaciones para la agricultura bajo riego.

\section{Año 2009}

El ciclo tardío del año 2009 se extendió entre el 9 de febrero y el 29 de mayo. El cultivo fue fertilizado a la plantación con $260 \mathrm{~kg}$ ha $^{-1}$ de 20-14-3 $(\mathrm{N}-\mathrm{P}-\mathrm{K})+\mathrm{Mg}$ y S. El esquema del ensayo consistió en diagramar sobre el lote una grilla rectangular de $5 \times 5$ sitios de muestreo separados cada $47 \mathrm{~m}$ en el sentido N-S y cada $44 \mathrm{~m}$ en el sentido de los surcos de riego (E-O). En cada sitio de aproximadamente $1 \mathrm{~m}^{2}$ de superficie se midió la fracción de radiación interceptada $(f R F A l)$ y la cobertura $(f)$ con una frecuencia media de 12 días.

Las determinaciones de fRFAl se realizaron utilizando un contador del flujo de fotones fotosintéticamente activos o "interceptómetro" (PAR/LAI Ceptometer, Decagon Devices, Pullman, Washington, USA), con la barra dispuesta de manera perpendicular al surco, en distintos lugares de cada estación de muestreo. Los valores de fRFAl se obtuvieron con la siguiente expresión:

$$
f R F A I=1-\left(\frac{F R F A_{\text {abajo }}}{F R F A_{\text {arriba }}}\right)
$$

siendo FRFA arriba $_{\text {la lectura del flujo instantáneo }}$ de radiación fotosintéticamente activa medida por

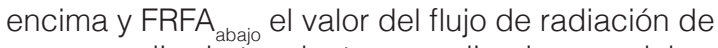
un promedio de tres lecturas realizadas por debajo del dosel. Las observaciones se realizaron sólo bajo condiciones de cielo despejado y durante las horas próximas al mediodía solar, lo que permitió homogeneizar las mediciones (de la Casa et al., 2007).

La fracción de suelo cubierto por papa ( $f$ ) se determinó a partir de una fotografía digital adquirida de modo vertical sobre el cultivo y técnicas de interpretación de imágenes (Rodríguez et al., 2000). El valor de $f$ se obtuvo por medio de un algoritmo de clasificación de imágenes digitales que aplica el método de máxima verosimilitud (Eastman, 1997). El mismo prevé la identificación de 4 clases: suelo y follaje, al sol y con sombra, respectivamente. Cada fotografía vertical se tomó a una altura de 1,2 m, aproximadamente en el mismo lugar donde se realizó la lectura del interceptómetro, con el surco entre los camellones centrales como eje longitudinal medio. También se procedió a estimar fRFAl a partir de $f$ (fRFAI_f), de acuerdo a la siguiente expresión (de la Casa et al., 2008):

$$
f R F A I_{-} f=1-(1-f)^{0,7}
$$

Los valores acumulados de $f$ se ponderaron respecto al tiempo transcurrido entre las mediciones para obtener la duración de la cobertura $\left(D_{-} f\right)$, de acuerdo a la siguiente expresión (Boyd et al., 2002):

$$
D_{-} f=\sum_{1}^{n-1}\left(\frac{\left(f_{n}+f_{n-1}\right)}{2}\right)\left(t_{n}-t_{n-1}\right)
$$

$t_{n}$ son los días desde plantación en que se determinó $f_{n}$, y $t_{n-1}$ son los días desde plantación de la muestra inmediata anterior.

De igual modo se procedió con fRFAl y fRFAl_ $f$ para obtener los valores acumulados respectivos. Al final del ciclo se realizó la cosecha de $1 \mathrm{~m}^{2}$ de 
la totalidad de tubérculos producidos en cada sitio de muestreo para expresar el rendimiento en peso fresco (PF, t ha-1).

\section{Año 2010}

El ciclo tardío del año 2010 se extendió entre el 16 de febrero y el 29 de mayo, y sus resultados sirvieron, en ausencia de enfermedades, para corroborar los del año anterior. El cultivo fue fertilizado a la plantación con $250 \mathrm{~kg} \mathrm{ha}^{-1}$ 20-17-3 (N-P-K) + Mg y S, y posteriormente con $100 \mathrm{~kg} \mathrm{ha}^{-1}$ de 22-0-0 $+\mathrm{S}$, Ca y Mg.

El esquema del ensayo consistió en diagramar sobre el lote una grilla de $3 \times 3$ sitios de muestreo separados cada $70 \mathrm{~m}$ en el sentido N-S y cada 70 $m$ en el sentido de los surcos de riego (E-O). En cada sitio se realizó un submuestreo adquiriendo nueve fotografías verticales en distintas posiciones equidistantes de aproximadamente $3 \mathrm{~m}^{2}$ de superficie (tomadas a 2,5 $\mathrm{m}$ de altura), para obtener el valor medio de cobertura ( $f$ ) por fecha con una frecuencia media de días similar al año anterior.

La cobertura se calculó, de acuerdo con Adamsen et al. (1999) y Gitelson et al. (2002), a partir de un procedimiento de análisis digital que determina la presencia de vegetación sobre el suelo apelando a la descomposición colorimétrica de la imagen en el rango del visible, y realizando en cada píxel un cociente entre las bandas verde y roja, siendo suelo cuando el cociente es menor a 1 y vegetación en caso contrario. En 2010, de la Casa et al. (2010) determinaron que los valores obtenidos con este procedimiento no difieren de los que produce el método de máxima verosimilitud y resulta más fácil de implementar ya que el cálculo es completamente automático.

\section{RESULTADOS Y DISCUSIÓN}

El ciclo 2009 de papa tardía presentó un ataque generalizado de trips (Frankliniella occidentalis Pergande), vector que transmitió la enfermedad de "top necrosis" (causada por el virus del marchitamiento del tomate-TSWV) (com. pers. Vilma Mazzuferi). En el lote analizado esta virosis se manifestó a los pocos días de la emergencia, de modo que la población de plantas resultó disminuida. Esta merma del stand de plantas redujo drásticamente la fracción de radiación interceptada en algunos sectores y, de acuerdo a la repercusión productiva de este factor (Kooman et al., 1996a; Caldiz et al., 2001), propició analizar la influencia de la cobertura sobre la productividad, como así también eva-
Iuar la variabilidad espacial del rendimiento en el lote. El desarrollo que experimentó la cobertura del follaje de papa a lo largo del ciclo para cada uno de los 25 sitios de la grilla de muestreo en 2009 se presenta en la Figura 1. La línea de guiones une las mediciones del sitio (1.4), donde en promedio la cobertura alcanza valores mínimos, y la continua hace lo propio en el sitio (5.1) que representa los máximos.

Las diferencias en los valores de cobertura son escasas hasta los 26 días posteriores a la plantación, presentando un rango máximo de $f$ entre las parcelas que no excede 10\%. Este rango se incrementa luego de manera progresiva hasta alcanzar una diferencia máxima (mayor a 60\%) a los 60 días de la plantación, para volver a disminuir hacia el final del ciclo. La Figura 1 permite apreciar también una caída generalizada de la tasa de crecimiento de la cobertura que acontece entre 30 y 40 días de la plantación, lo que pone en evidencia la influencia generalizada que tuvo la peste negra sobre el cultivo.

Previo a considerar la situación de la variabilidad productiva del lote, interesa analizar la relación entre fRFAly $f$, a los efectos de evaluar en particular el alcance explicativo de la variable que se obtiene a partir de fotografías digitales. Bajo condiciones experimentales caracterizadas por una merma ostensible en la superficie foliar, la Figura 2 presenta las relaciones entre los valores observados de fRFAl y los que se estiman a partir de la información de cobertura, utilizando directamente $f$ como sucedáneo, y también con el valor obtenido de la ecuación (2) (de la Casa et al., 2008). Los datos analizados corresponden a un promedio de las mediciones que se realizaron hasta 60 días posteriores a la plantación, momento en que el lote presentó la máxima diferencia de fentre parcelas y anterior al comienzo de la senescencia. Las funciones lineales de ajuste muestran que la utilización directa de $f$ produce una sistemática sobreestimación de fRFAl en todo el rango analizado, con una raíz del error cuadrático medio (RMSE) de 0,06, lo que debe ser tenido en cuenta si se pretende estimar la cantidad de radiación interceptada apelando a esta medición.

Por su parte, el modelo que estima fRFAl a partir de $f$ (de la Casa et al., 2008) si bien produce una ligera subestimación, en particular para valores altos, da lugar a una curva de ajuste más próxima a la recta identidad respecto de la que se obtiene a partir de $f$, que se refleja en la reducción del RMSE a la mitad. No obstante, la capacidad para explicar la variabilidad de fRFAl en ambos casos es de $88 \%$ $(P<0,01) y$, de una u otra forma, se aprecia que $f$ representa de manera muy ajustada la variación de 


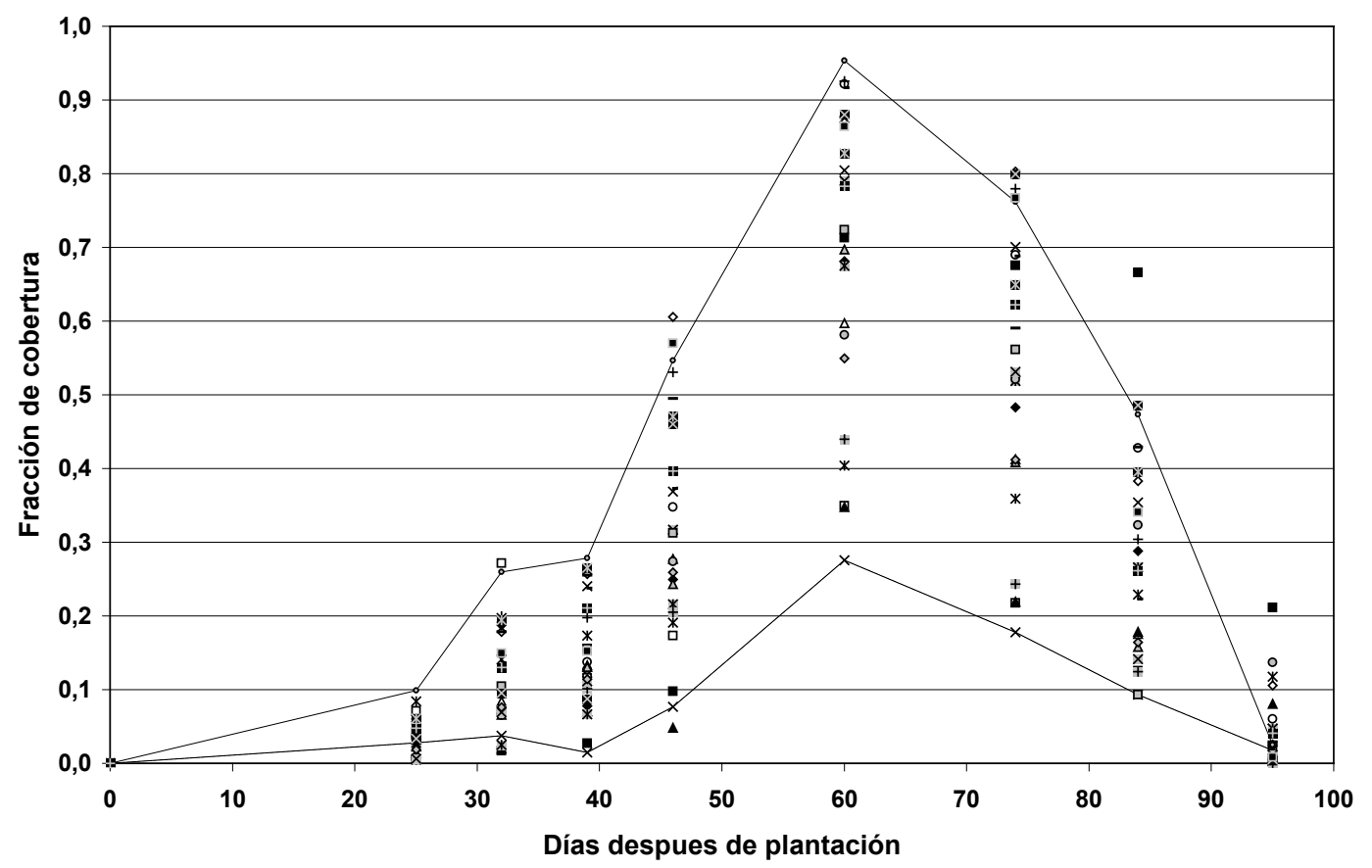

\begin{tabular}{|c|c|c|c|c|c|c|c|c|c|c|c|c|c|c|c|c|c|c|}
\hline - (1.1) & 口 & $(1.2)$ & $\Delta$ & (1.3) & $x$ & (1.4) & $*$ & (1.5) & o & $(2.1)$ & + & $(2.2)$ & - & (2.3) & - & (2.4) & $\diamond$ & (2.5) \\
\hline - (3.1) & $\Delta$ & (3.2) & $x$ & (3.3) & * & (3.4) & $\circ$ & (3.5) & + & (4.1) & $\Delta$ & $(4.2)$ & $x$ & (4.3) & $\diamond$ & (4.4) & $\square$ & (4.5) \\
\hline$\multimap(5.1)$ & 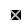 & (5.2) & $w$ & (5.3) & - & (5.4) & : & (5.5) & & & & & & & & & & \\
\hline
\end{tabular}

Figura 1. Evolución de la fracción de cobertura (f) de papa en los 25 sitios del lote del cinturón verde de Córdoba durante el ciclo tardío 2009 .

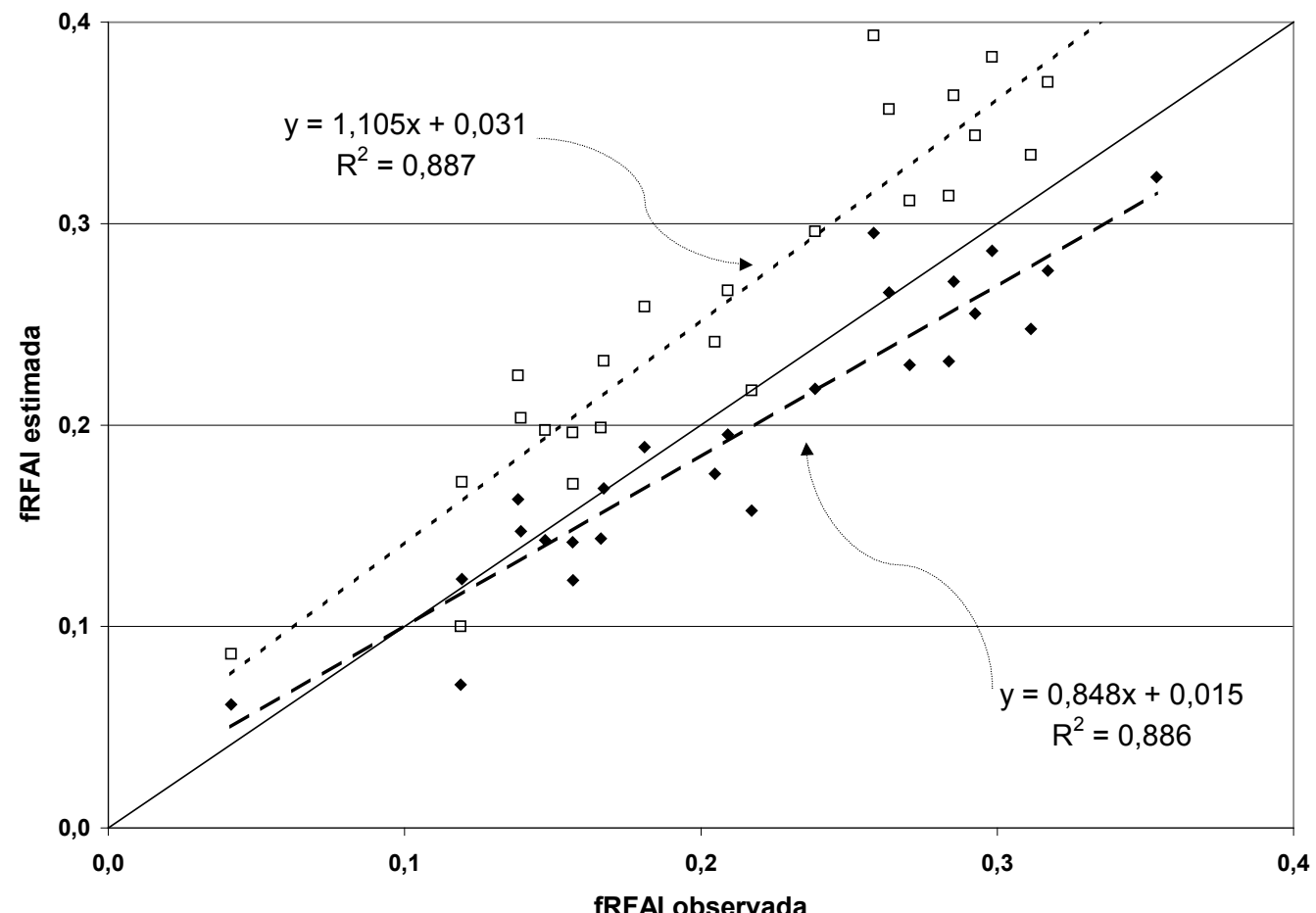

Figura 2. Relaciones entre el valor medio de la fRFAl observada, y los valores estimados directamente a partir de la cobertura ( $f$ ) (guiones cortos) y con el modelo fRFAI_f(guiones largos). 
fRFAl en el lote. En consecuencia, se puede asumir que en los sectores donde la magnitud de f resultó más elevada, mayor fue la cantidad de radiación interceptada y más alta la capacidad productiva potencial (Kooman et al., 1996a; Caldiz et al., 1999; Kadaja y Tooming, 2004; de la Casa et al., 2011).

La diferencia marcada que presentó $f$ en los distintos sectores del lote se puede aprovechar para analizar su repercusión productiva, tanto de manera acumulativa como para cada fecha en particular. En el primer caso, se utilizó la duración de la cobertura de los primeros 60 días del ciclo como variable independiente (hasta alcanzar el valor máximo de la diferencia entre sitios) y, en el segundo, se emplearon sólo las mediciones de $f$ realizadas a los 60 días de plantación (10 de abril), fecha en que se registró la diferencia máxima (Figura 1). Los resultados de la relación lineal entre el rendimiento de tubérculo en fresco y las distintas variables independientes utilizadas se presentan en la Tabla 1.

Las mediciones de fRFA/ son las que presentan mayor capacidad explicativa de la variabilidad del rendimiento en el lote, de modo tal que su valor acumulativo ponderado por el tiempo, hasta que el cultivo alcanza la máxima cobertura, resulta en un coeficiente de determinación significativo de aproximadamente 0,86 . En caso de utilizar directamente valores acumulados de $f$ o fRFAl_f para explicar la variabilidad espacial del rendimiento, si bien el $\mathrm{R}^{2}$ resulta un tanto menor que al emplear $D_{-} f R F A l$, la variabilidad residual no se incrementa demasiado. El análisis de la variabilidad espacial del rendimiento utilizando información recabada sólo el día de máxima diferencia de cobertura, produce niveles de determinación un poco más reducidos en los tres casos y mantiene un $\mathrm{R}^{2}$ mas elevado para las determinaciones a partir del interceptómetro.

\section{Análisis por filas y columnas}

La diferencia que presentó la evolución de la cobertura en distintos sectores del lote se puede proyectar para explicar la heterogeneidad productiva del cultivo de papa. La estructura de filas (surcos) y columnas de la grilla de muestreo resulta apropiada para realizar un análisis territorial tendiente a determinar si los valores de rendimiento en el lote responden a una distribución aleatoria o, por el contrario, presentan alguna dependencia espacial. Para responder a este interrogante la Figura 3 presenta la relación entre la cobertura y el rendimiento de tubérculo, correspondientes a un valor medio por fila y por columna, para el día de máxima diferencia de cobertura.

De acuerdo a la Figura 3, los resultados del ensayo en este lote de producción comercial de papa tardía de 2009 muestran que el rendimiento del cultivo presenta una dependencia espacial ligada principalmente a las diferencias entre las filas del ensayo, donde la relación con la cobertura es significativa $(P<0,01)$, y no así cuando se analiza el comportamiento entre columnas. Mientras el rango de variación de $f$ entre filas alcanza $40 \%$, entre columnas la diferencia sólo es de 15\%. Este comportamiento espacial se puede explicar en razón del manejo del cultivo en camellones, ligado a labores culturales que se realizan principalmente en el sentido de los surcos: plantación, aporques, tratamientos terapéuticos preventivos. Para este año en particular, el desarrollo de la peste negra también puede haber contribuido a este comportamiento, ya que el avance de la enfermedad se presentó de sur a norte, es decir de manera paralela a las filas de plantas.

Si bien en este ensayo se destaca la influencia significativa de las filas en la estructura de variabilidad del rendimiento de papa, no se puede soslayar que existe una gran variabilidad local, lo que se pone en evidencia en los valores elevados que adopta el desvío estándar (Figura 3). En este sentido se considera que los valores obtenidos, más allá de la variabilidad sistemática del rendimiento de papa que está ligada de un modo inevitable a las operaciones y tareas culturales, muestran un comportamiento predominantemente aleatorio,

Tabla 1. Coeficientes de regresión (a: ordenada al origen; b: pendiente) y determinación $\left(R^{2}\right)$ entre el rendimiento de tubérculo en peso fresco $\left(\mathrm{t} \mathrm{ha} \mathrm{H}^{-1}\right.$ ) y variables independientes: fracción de radiación fotosintéticamente activa interceptada ( $\left.f R F A l\right)$ y su duración ( $\left.D \_f R F A l\right)$, cobertura $(f)$ y su duración $\left(D_{-} f\right)$ y el valor estimado de $f R F A /$ a partir de $f(f R F A I f f)$, y su duración ( $\left.D \_f R F A I f\right)$, de acuerdo a diferentes criterios temporales en el año 2009. Todas las relaciones son estadísticamente muy significativas $(P<0,00001)$.

\begin{tabular}{|c|c|c|c|c|c|c|}
\hline \multirow[b]{2}{*}{ Coeficientes } & \multicolumn{3}{|c|}{ Hasta 60 días después de la emergencia } & \multicolumn{3}{|c|}{ Día del rango máximo de cobertura $(10 / 04)$} \\
\hline & D_fRFAI & $D \_f$ & $D \_f R F A I \_f$ & fRFAI & $f$ & fRFAl_f \\
\hline $\mathbf{a}$ & $-1,122$ & $-0,762$ & 0,599 & 2,107 & $-3,840$ & $-0,579$ \\
\hline b & 2,115 & 1,689 & 1,992 & 31,451 & 30,576 & 30,759 \\
\hline $\mathbf{R}^{2}$ & 0,858 & 0,768 & 0,767 & 0,777 & 0,705 & 0,706 \\
\hline
\end{tabular}




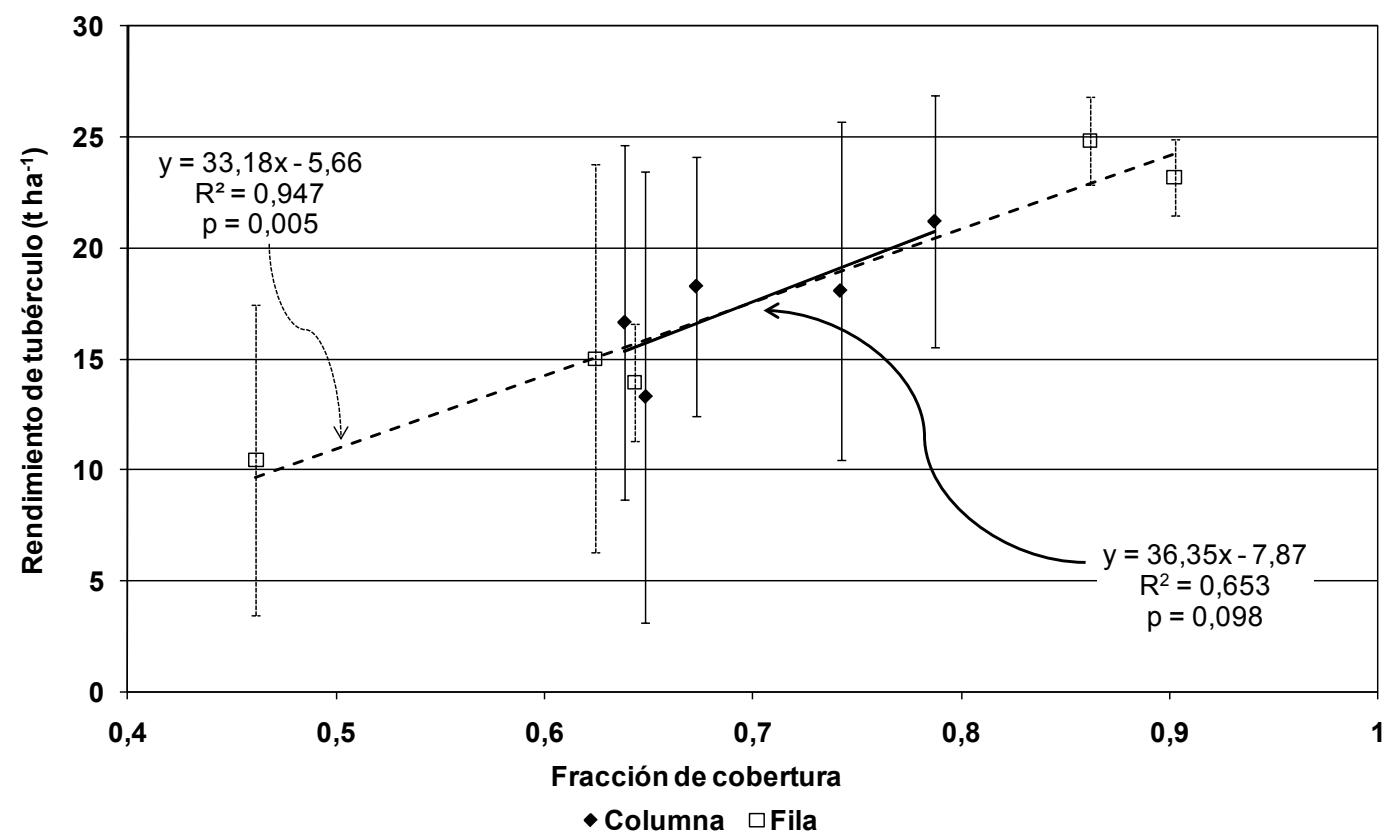

Figura 3. Relación entre el valor medio de la fracción de cobertura por columna (rombo relleno) y por fila (cuadrado vacío) y el rendimiento de papa (PF) a los 60 días después de plantación (10/04) del ciclo tardío 2009 en Córdoba. La barra expresa \pm el desvío estándar sobre el valor medio y p es el valor de probabilidad calculado.

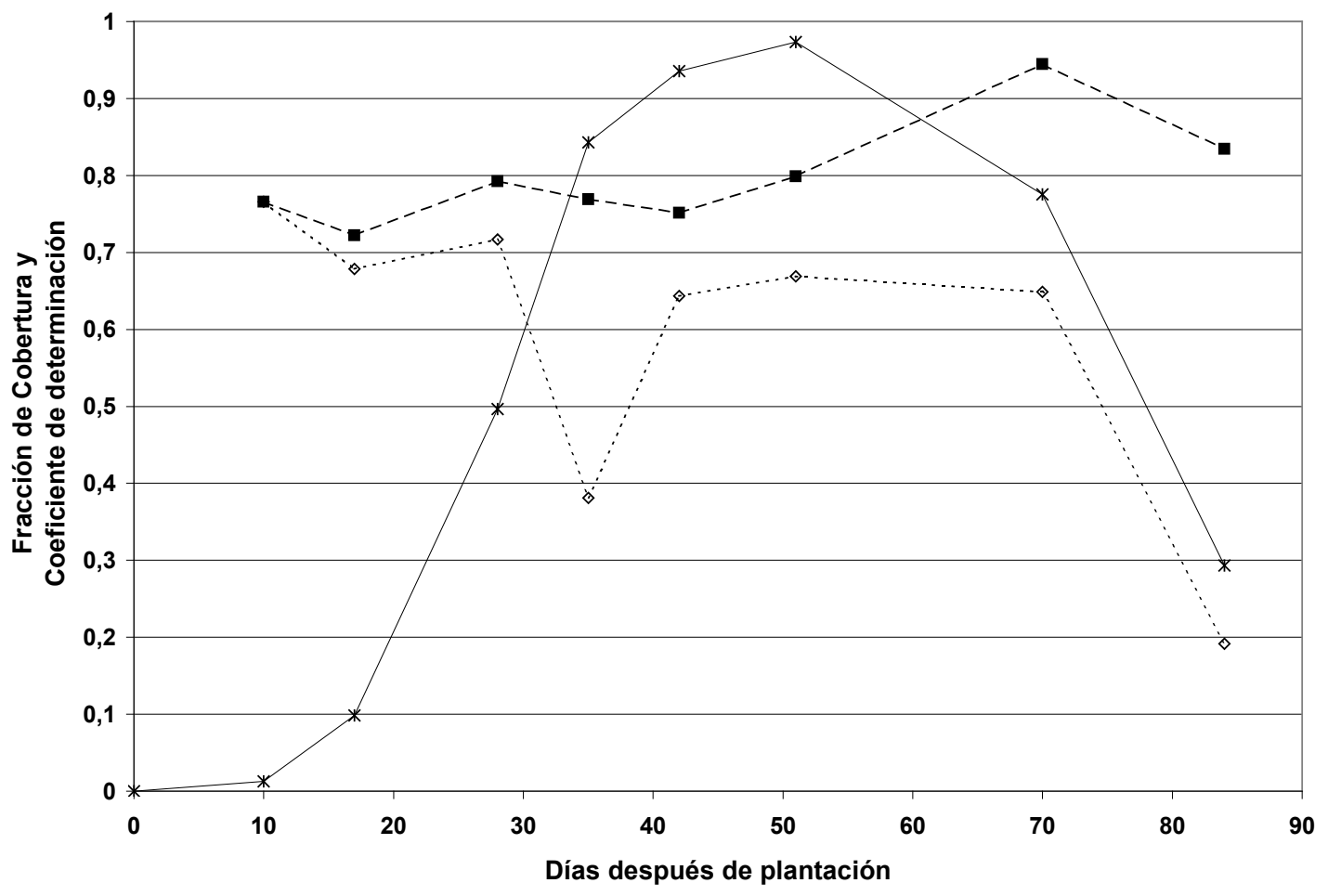

$-. \diamond . \cdot R 2 \quad$ (f y rendimiento) - - - R2 (D_f y rendimiento) $\quad *$ Fracción de cobertura

Figura 4. Valor medio de la fracción de cobertura de papa y coeficientes de determinación $\left(R^{2}\right)$ entre la fracción de cobertura $(f)$ y la duración de la cobertura $\left(D_{-} f\right)$ y el rendimiento de tubérculo a cosecha, respeto a los días después de plantación durante el ciclo tardío 2010 en Córdoba. 
que no justificaría implementar un manejo localizado.

En el año 2010 se realizó otro muestreo en el mismo lote de producción, tendiente a corroborar la capacidad predictiva de la cobertura bajo otras condiciones ambientales. Para poder evaluar mejor la variabilidad local en el lote se incrementó el tamaño de las parcelas, de modo que se realizaron mediciones más intensivas en cada sector, pero reduciendo la grilla de exploración. El cultivo este año prosperó en forma vigorosa y sin inconvenientes de tipo sanitario, como muestra la Figura 4, por lo que los valores máximos de la fracción de cobertura a los 51 días después de plantación (8 de abril) presentaron un rango estrecho entre 93 y 99 $\%$ entre las distintas parcelas. La Figura 4 presenta en distintos momentos del ciclo la variación que alcanza el coeficiente de determinación para la relación lineal entre los valores de la duración de cobertura $\left(D_{-} f\right)$ hasta ese momento y el rendimiento de tubérculo a cosecha. También se presenta el $R^{2}$ que se obtiene a partir de la fracción de cobertura observada cada fecha de manera independiente.

La Figura 4 pone claramente de manifiesto que el valor de cobertura acumulada $\left(D_{-} f\right)$ hasta cada fecha del muestreo presenta una buena capacidad predictiva de la productividad, con coeficientes de determinación para la relación lineal siempre superiores a $0,70(P<0,01)$, hasta alcanzar un valor máximo que supera 0,94 a los 70 días de la plantación, prácticamente un mes antes de la cosecha del cultivo. La aptitud predictiva de $D_{-} f$ sobre el rendimiento se puso rápidamente en evidencia durante el ciclo de producción 2010, ya que a partir del primer muestreo realizado a los 10 días de plantación (26 de febrero), y para un rango de la condición de cobertura media entre sectores de 0,77\% (sector 33) y 1,55\% (sector 12), se pudo establecer una relación lineal significativa que se incrementa a medida que transcurre el ciclo de producción. Por su parte, el valor de cobertura observada cada fecha en particular presenta una capacidad predictiva del rendimiento a cosecha inferior, pero del mismo orden de magnitud $\left(R^{2}=0,67\right.$ a los 51 días de la plantación) que el año anterior. Cuando se aproxima el final el ciclo y con el deterioro de la parte área como consecuencia de la senescencia del cultivo, la cobertura pierde esta capacidad.

La cobertura del follaje y su duración han sido evaluados ya por otros autores como indicadores de la potencialidad productiva en papa, para analizar a nivel regional el comportamiento de distintos ambientes (sitios y ciclos) y genotipos (Kooman et al., 1996a; Caldiz et al., 1999). Boyd et al. (2002), por su parte, al obtener correlaciones elevadas entre $f$ y el índice de área foliar (IAF) bajo diferen- tes prácticas de manejo y niveles de competencia extremos, muestran que la primera de ellas puede alcanzar un rango predictivo similar a la segunda, argumento que se sostiene en mayor medida al incrementarse la correlación entre la cobertura del follaje y la fRFAl obtenida por la ley de LambertBeer a partir del IAF. También determinaron que la duración de la cobertura explica en igual o mayor proporción la variabilidad del rendimiento de tubérculo que la duración de IAF. De acuerdo a la Figura 4, la estimación de la productividad a partir de la cobertura mantiene su potencial predictivo hasta que el cultivo reduce su valor por debajo de $50 \%$. Esto confirma la validez del criterio de Kooman et al. (1996a) para establecer la finalización del ciclo de crecimiento en papa, porque cuando la intercepción de la radiación disminuye por debajo de $50 \%$ no se produce substancialmente nuevo aporte de asimilados a los órganos de cosecha.

La apropiada capacidad predictiva que mostró la cobertura del follaje, bajo las condiciones ambientales y manejo técnico que predominan en el cinturón verde de Córdoba durante el ciclo de papa tardía, es probable que no se exprese de igual modo bajo otras condiciones de producción que permitan ciclos de cultivo más extendidos o densidades de plantación más elevadas. En este sentido, Firman \& Allen (1989) señalan que aunque la cobertura del follaje resulta útil para evaluar el crecimiento de la papa, no es completamente adecuada para estimar la intercepción de la luz y puede conducir a errores en el cálculo de la eficiencia de conversión de materia seca.

Los valores de cobertura, que son determinaciones operativamente sencillas de obtener a partir de un equipamiento fácilmente disponible y de bajo costo (cámara digital), confirmaron asimismo su utilidad para identificar de manera temprana los sitios que presentan limitaciones productivas en el lote de producción comercial de papa. Esta información resulta de interés a los efectos de su empleo como elemento de diagnóstico que permita distinguir zonas de manejo localizado en un lote y puede configurar un procedimiento alternativo para el análisis de la variabilidad espacial (Hornung et al., 2006).

\section{CONCLUSIONES}

La determinación de la fracción de suelo cubierto por el follaje constituye una técnica muy accesible para realizar el seguimiento del cultivo de papa a lo largo del ciclo y, debido a su relación estrecha con la fracción de radiación fotosintéticamente activa interceptada, también permite 
estimar el rendimiento de manera anticipada a la cosecha con una precisión aceptable.

La duración de la cobertura obtenida alrededor de los valores máximos (entre 50 y 70 días de la plantación) mostró un comportamiento predictivo aceptable, de modo que la relación lineal con el rendimiento de tubérculos a cosecha arrojó un $\mathrm{R}^{2}$ de 0,77 el año 2009, a partir de un muestreo menos intensivo, que aumentó a 0,94 en 2010 cuando la relación se estableció utilizando valores medios de distintos sitios del mismo sector/nodo.

La fracción de cobertura también resultó de utilidad para conocer la variabilidad espacial del rendimiento de manera anticipada, a los efectos de establecer un eventual manejo localizado o de tasa variable.

\section{BIBLIOGRAFÍA}

Adamsen, F.J., P.J. Pinter, Jr., E.M. Barnes, R.L. La Morte, G.W. Wall, S.W. Leavitt and B.A. Kimball, 1999. Measuring wheat senescence with a digital camera. Crop Sci. 39:719-724.

Boyd, N.S., R. Gordon and R.C. Martin, 2002. Relationship between leaf area index and ground cover in potato under different management conditions. Pot. Res. 45:117-129.

Caldiz, D.O., O.H. Caso, G. Vater and L.V. Fernández, 1999. The potential for production of high quality seed potatoes in Tierra del Fuego Island, Argentina. Pot. Res. 42:9-23.

Caldiz, D.O., F.J. Gaspari, A.J. Haverkort and P.C. Struik, 2001. Agro-ecological zoning and potential yield of single or double cropping of potato in Argentina. Agric. For. Meteorol. 109:311-320.

de la Casa, A., G. Ovando, A. Rodríguez, L. Bressanini y E. Buffa, 2003. Evaluación espacial del nitrógeno disponible en un cultivo de papa (Solanum tuberosum $L$. cv. Spunta) en Córdoba, Argentina. Agricultura Técnica (Chile) 63:311-318.

de la Casa, A., G. Ovando, L. Bressanini, Á. Rodríguez y J. Martínez, 2007. Uso del índice de área foliar y del porcentaje de cobertura del suelo para estimar la radiación interceptada en papa. Agricultura Técnica (Chile) 67:78-85.

de la Casa, A., G. Ovando, L. Bressanini, Á. Rodríguez y J. Martínez, 2008. Evaluación de un modelo para estimar la fracción de radiación interceptada en papa (Solanum tuberosum L.) bajo distintas densidades de plantación. XII Reunión Argentina de Agrometeorología. Compilado por Luis G. Buitrago - $1^{\text {a }}$ Ed. - San Salvador de Jujuy - Universitaria de Jujuy: Univ. Nacional de Jujuy: Fac. de Ciencias Agrarias, 2008. ISBN 978-

\section{0-721-320-5}

de la Casa, A., G. Ovando, L. Bressanini, Á. Rodríguez y J. Martínez, 2010. Determinación de la fracción de suelo cubierta con el follaje de papa a partir del cociente entre bandas de fotografías digitales. Actas de la XIII Reunión Argentina y VI Latinoamericana de Agrometeorología. Bahía Blanca, Buenos Aires, Argentina. 20 al 22 de octubre de 2010. ISBN 978-98726317-0-3.

de la Casa, A., G. Ovando, L. Bressanini, J. Martínez y Á. Rodríguez, 2011. Eficiencia en el uso de la radiación en papa estimada a partir de la cobertura del follaje. Agriscientia 26:21-30.

Eastman, R.J., 1997. Clasification of remotely sensed imagery. In: IDRISI: User's guide Idrisi for Windows, Worcester, MA, USA, Clark University.

Evett, S.R. and J.A. Tolk, 2009. Introduction: Can water use efficiency be modeled well enough to impact crop management? Agron. J. 101:423-425.

Firman, D.M., and E.J. Allen, 1989. Relationship between light interception, ground cover and leaf area index in potatoes. The Journal of Agricultural Science 113:355-359.

Gitelson, A.A., Y.J. Kaufman, R. Stark and D. Rundquist, 2002. Novel algorithms for remote estimation of vegetation fraction. Remote Sens. Environ. 80:76-87.

Hornung, A., R. Khosla, R. Reich, D. Inman and D.G. Westfall, 2006. Comparison of site-specific management zones: soil-color-based and yield-based. Agron. J. 98:407-415.

Jarsún, B., J. Gorgas, E. Zamora, H. Bosnero, E. Lovera, A. Ravelo y J. Tassile, 2006. Los suelos de Córdoba. Agencia Córdoba Ambiente e Instituto Nacional de Tecnología Agropecuaria, EEA Manfredi. Córdoba, Argentina.

Kadaja, J. and H. Tooming, 2004. Potato production model based on principle of maximum plant productivity. Agric. For. Meteorol. 127:17-33.

Kooman, P.L., M. Fahem, P. Tegera and A.J. Haverkort, 1996a. Effects of climate on different potato genotypes. 1. Radiation interception, total and tuber dry matter production. Eur. J. Agron. 5:193-205.

Kooman, P.L., M. Fahem, P. Tegera and A.J. Haverkort, 1996b. Effects of climate on different potato genotypes. 2. Dry matter allocation and duration of the growth cycle. Eur. J. Agron. 5:207-217.

Moran, M.S., Y. Inoue and E.M. Barnes, 1997. Opportunities and limitations for image-based remote sensing in precision crop management. Remote Sens. Environ. 61:319-346.

Pierce, F.J. and P. Nowak, 1999. Aspects of precision agriculture. Advances in Agronomy 67:1-85.

Purcell, L.C. 2000. Soybean canopy coverage and light 
interception measurements using digital imagery. Crop Science 40:834-837.

Rodríguez, A., A. de la Casa, R. Accietto, L. Bressanini y G. Ovando, 2000. Determinación del área foliar en papa (Solanum tuberosum L., var.Spunta) por medio de fotografías digitales conociendo la relación entre el número de pixeles y la altura de adquisición. Rev. Bras. Agrometeorol. 8:215-221.

Stanhill, G., 1986. Water use efficiency. Advances in Agronomy 39:53-85.
Steduto, P., T.C. Hsiao, D. Raes and E. Fereres, 2009. AquaCrop-The FAO crop model for predicting yield response to water: I. Concepts and underlying principles. Agron. J. 101:426-437.

Steven, M.D., P.V. Biscoe, K.W. Jaggard and J. Paruntu, 1986. Foliage cover and radiation interception. Field Crop Res. 13:75-87.

Van Oijen, M., 1991. Light use efficiencies of potato cultivars with late blight (Phytophthora infestans). Pot. Res. 34:123-132. 